\title{
La vivienda y la sustentabilidad en la Riviera Maya: los desbordes del turismo
}

\author{
Dwellings and sustainability in Riviera \\ Maya: the overflows of tourism
}

\author{
Paola Bagnera* y Belén Pennisi** \\ Fecha de recepción: 27-04-2014 - Fecha de aceptación: 10-02-2015 \\ Hábitat y Sociedad (ISSN 2173-125X), n. . 8, noviembre de 2015, pp. 97-112.
}

\section{Summary}

Access to urban land of the tourist 1cities 2towns of the Mexican Caribbean demonstrates an 1unequal 2unfair and fragmented configuration where social housing embodies responses that, with uncertain degree of sustainability, reproduces inequitable conditions on the exercise of the right to the town.

This study presents the results of the recognition of this particular production of residential borders in localities of Quintana Roo, Mexico: Cozumel, Playa del Carmen and Tulúm, by taking into account not only the physical materialization and urban configuration but also its social value.

These dwellings involve comprehensive recognition that is based on the hypothesis that all manifestations of urban precariousness imply an unsustainable expression in its configuration: as can be observed in the Mayan coastal habitat.

\section{Key words}

Sustainability, Urban habitat, Working-class Habitat, Tourism, Urban Overflows

\section{Resumen}

El acceso al suelo urbano en las ciudades turísticas del Caribe mexicano evidencia una configuración desigual y fragmentaria, donde la vivienda social materializa respuestas que, con diverso grado de sostenibilidad, reproduce condiciones inequitativas en el ejercicio del derecho a la ciudad.

El trabajo se centra en la presentación de los resultados del reconocimiento de esa particular producción de bordes residenciales en localidades de Quintana Roo, México: Cozumel, Playa del Carmen y Tulúm, atendiendo tanto a su materialización física y configuración urbana cuanto a su valoración social.

Estas manifestaciones habitacionales implican un reconocimiento integral, que parte de la hipótesis de que toda manifestación de precariedad urbana lleva implícita una expresión de insustentabilidad en su configuración, hecho que resulta evidenciado en el hábitat costero maya.

\section{Pallabras clave}

Sustentabilidad, Hábitat urbano, Hábitat popular, Turismo, Desbordes urbanos

\footnotetext{
Arquitecta en Secretaria Nacional de Acceso al Hábitat, Argentina.

** Proyectos IAPOS (Instituto Autárquico Provincial de Obra Social), Universidad Nacional del Litoral, Facultad de Arquitectura, Diseño y Urbanismo, FADU UNL, Argentina.
}

\section{Introducción}

El entorno paisajístico y ambiental de la Riviera Maya remite a valoraciones generadas por unas condiciones geográficas que son promovidas con una finalidad turística, del ocio y el disfrute. Sin embargo, dichas manifestaciones conllevan la particular circunstancia de evidenciar la fragmentación social y habitacional, que contrasta este idílico paisaje turístico respecto de las manifestaciones residenciales que configuran el hábitat de su población permanente. Los bordes urbanos se constituyen en el soporte básico de esta segregación urbana manifiesta: hacia la costa se concentran los centros hoteleros, recreativos, comerciales que organizan la oferta turística de la Riviera, mientras que son los bordes interio- 
res en sus expansiones territoriales los que conforman el soporte del hábitat popular.

Las caracterizaciones e indagaciones presentadas en el artículo, junto a sus conclusiones preliminares, se enmarcan en el proyecto de investigación "Indicadores de sustentabilidad aplicados a producciones de interés regional: monitoreo del hábitat urbano y definición de lineamientos para su producción sustentable. Los casos de Entre Ríos-Santa Fe, Argentina y Riviera Maya-Cozumel, México", que surge en el marco de cooperación bilateral entre el Ministerio de Ciencia y Tecnología argentino y su par, el Consejo Nacional de Ciencia y Tecnología mexicana. El proyecto problematiza en torno a la definición y aplicación de indicadores de sustentabilidad y su aplicación en diversas manifestaciones habitacionales en territorio de las universidades involucradas (UNL, Santa Fe-UQROO, Cozumel), atendiendo tanto a producciones de vivienda social -en el marco de políticas públicas o de acciones descentralizadas-, cuanto de situaciones de producción social del hábitat en ambos contextos, a partir de la hipótetica consideración de que la precariedad urbano-habitacional se evidencia tanto en procesos de producción social del hábitat cuanto en prácticas generadas a partir de lógicas públicas de intervención. En dicho marco, inferimos diversas relaciones que se reconocen en las preguntas de partida de la investigación, y que se orientan en torno a la relación que se produce entre dichas configuraciones - la "formal" y la "informal" - y la evidencia de la idea de sustentabilidad, a partir de una noción amplia e integral de la misma.

\section{Hábitat y Sustentabilidad: abordaje teórico y metodológico}

El desarrollo sustentable como es entendido como el "desarrollo económico y social y el aprovechamiento de los recursos naturales realizado a través de una gestión apropiada del ambiente, de manera tal, que no comprometa las posibilidades de las generaciones presentes y futuras", se reconoce en la misma una consideración amplia, incluyendo integralmente aspectos sociales, económicos y ambientales, conformándose en el eje de abordaje de la noción de sustentabilidad en años recientes en la región. La Conferencia de Naciones Unidas para los Asentamientos Humanos (Hábitat II, 1996) se constituyó en un momento significativo para la incorporación de nuevas lógicas vinculadas a la planificación y gestión de los asentamientos humanos, así como los propósitos relativos a la reducción de la pobreza. Estos elementos — habitualmente asociados a la idea de habitabilidad - dan cuenta de una noción que privilegia la preocupación por una redistribución y desarrollo equitativo de variables económicas, sociales y ambientales que definen la mencionada noción de sustentabilidad.

La aproximación metodológica se basa en el análisis comparado de áreas de estudio que evidencian situaciones disímiles, pero que adquieren cierto grado de proximidad tanto en sus manifestaciones territoriales como en su condición de bordes, entendiéndolos como manifestación particularizada del hábitat ligado muchas veces a condiciones de precariedad urbano-habitacional, exacerbados por la presencia de un territorio vulnerable y expuesto al riesgo. $\mathrm{El}$ análisis de dichas manifestaciones se desarrolla a partir de la definición de indicadores de sustentabilidad para el reconocimiento y evaluación de la precariedad urbana a partir del hipotético planteo de que toda manifestación de 
precariedad urbana lleva implícita una expresión de insustentabilidad en su configuración, o expresada en sentido inverso y atendiendo a una resultante proyectual de la indagación: el mejoramiento de las condiciones de precariedad conlleva una mayor sustentabilidad en la configuración del hábitat.

En este sentido, la noción de "sustentabilidad" es entendida en un sentido amplio, superando la mera consideración ambiental para estar más ligada a evidenciar si existen condiciones de equidad, equilibrio o paridad entre los aspectos sociales, económicos, institucionales y urbano-ambientales, que permiten su reconocimiento. Esta consideración implica que la metodología planteada reconoce la definición de indicadores que se enmarcan en subsistemas o dimensiones que refieren a dicha integralidad:

- La Dimensión Ambiental, Territorial y Urbano-Arquitectónica: es una dimensión que persigue la intención de analizar la cualificación del hábitat, dada a partir de la interacción de sus aspectos naturales (ambientales) y construidos (territoriales y urbanos, atendiendo a incluir la resolución habitacional en sus aspectos materiales constructivos).

- La Dimensión Social: se propone analizar la calidad de vida de la población (respecto de la satisfacción de necesidades materiales y no materiales).

- La Dimensión Económica: plantea abordar los medios para la subsistencia familiar y los modos de acceso financiero a una vivienda digna.

- La Dimensión Institucional: evidenciada por los mecanismos de democratización de la relación entre los ciudadanos y sus demandas (o derechos).

Los diversos antecedentes utilizados para la estructuración de las variables e indicadores de análisis, dan cuenta de la complejidad en su abordaje. En este sentido, resulta orientadora la meta 11 (Objetivos Desarrollo del Milenio), configurándose como una de las metas del Objetivo $7^{1}$ y persiguiendo la mejora de la calidad de vida de los habitantes de los asentamientos precarios, siendo acompañada por la incorporación de los principios del desarrollo sostenible en las políticas y los programas nacionales, invirtiendo la pérdida de recursos del medio ambiente, así como por la reducción a la mitad, para el año 2015, del porcentaje de personas que carecen de acceso sostenible a agua potable y al saneamiento.

Si bien las fuentes utilizadas en la indagación fueron de diverso tipo (censales, cartográficas, informes oficiales, etc.), prevalecen en esta presentación los datos provenientes de fuentes orales y materiales producto del relevamiento desarrollado por el equipo de trabajo. Se considerarán como fuentes orales las relevadas a partir de informantes calificados (habitantes, funcionarios, técnicos, etc.); mientras que las fuentes materiales refieren al estudio y caracterización urbano-habitacional de las áreas identificadas a partir del rastreo in situ de sus manifestaciones espaciales. El objeto de estudio reviste la particularidad de constituirse habitualmente en un "área inexplorada" por los datos y registros oficiales, razón por la cual las dos últimas fuentes no revisten el carácter de "complementarias", sino que asumen un rol específico en el reconocimiento de las manifestaciones del hábitat precario.

El análisis comparado implica la lectura y análisis conclusivo para el
1 La lectura a partir de las metas del Objetivo 7, se toman (entre otras) como referencia para el análisis a partir de la presunción mencionada de que las condiciones de precariedad urbano-habitacional refieren a una integralidad que puede estar manifestándose tanto en urbanizaciones espontáneas o de producción social del hábitat, cuanto planificadas como ejercicio de políticas públicas, cuando estas abordan en forma insuficiente el problema que pretenden resolver, intensificando muchas veces sus impactos y condiciones resultantes. 
reconocimiento de las características y problemáticas específicas de las áreas analizadas. Este proceso permite el reconocimiento de desigualdades sociales, materializadas en el territorio como un aporte a la generación de políticas urbanas inclusivas e intervenciones de recualificación y mejoramiento urbano de las áreas consideradas.

\section{Los casos de estudio: Cozumel, Playa del Carmen y Tulúm}

El trabajo que presentamos, recorta y centra su consideración a las manifestaciones mexicanas de la cuestión, atendiendo particularmente a la lectura de uno de los objetivos del milenio (el derecho a la vivienda adecuada $)^{2}$ y su lectura a través de los mencionados subsistemas o dimensiones.

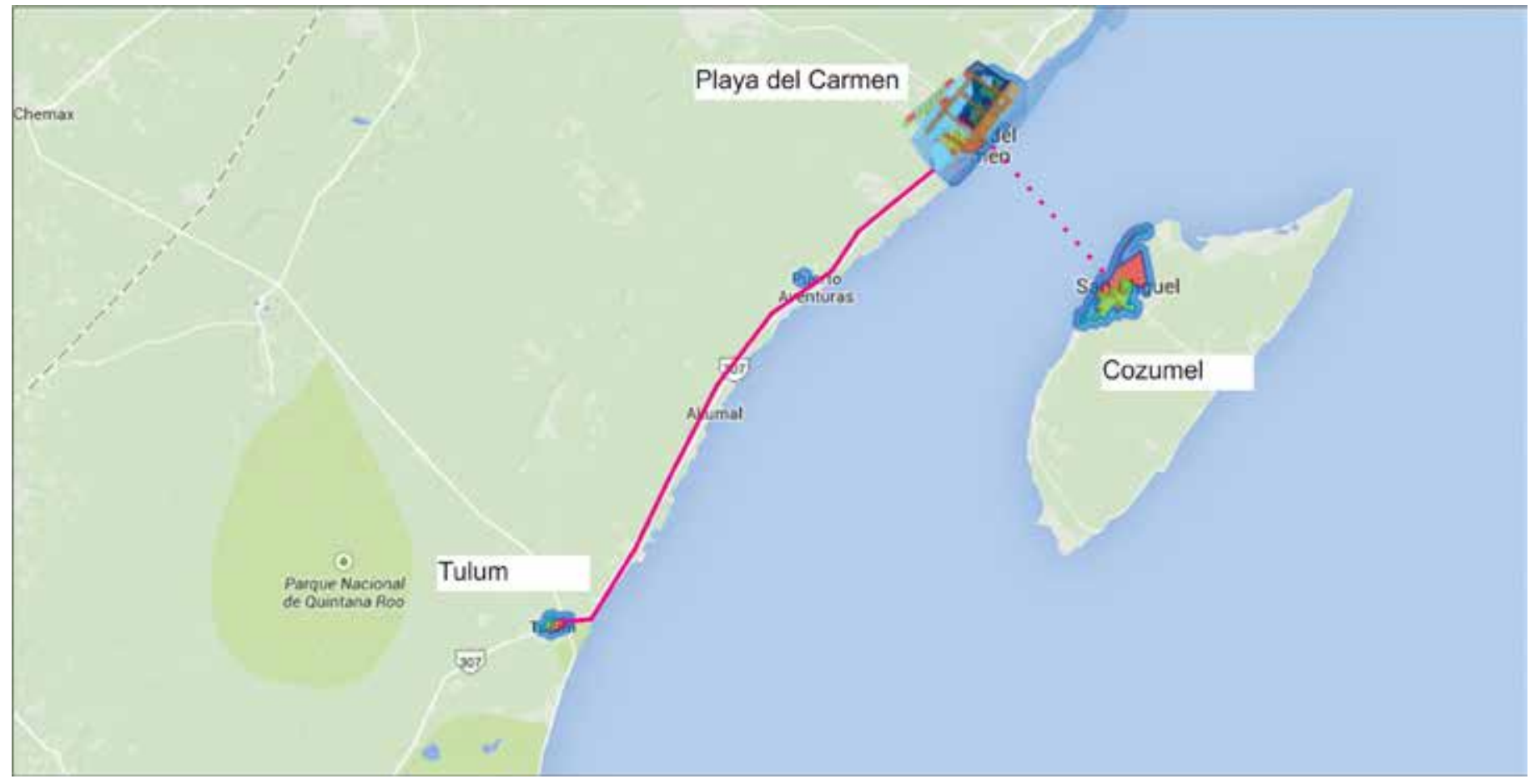

Figura 1. Localización de los casos de estudio. Fuente: SIG (Sistema de Información Geográfica, México). Usos del suelo http://sig.ruv.org.mx/.
2 Los objetivos del milenio, y la particular referencia al derecho a la vivienda adecuada, se consideran protagónicos en el abordaje de la cuestión, en términos del reconocimiento de la problemática y de su caracterización específica.
El reconocimiento de dichos indicadores se desarrolla en las localidades de Cozumel, Playa del Carmen y Tulúm, insertas en el Estado de Quintana Roo y formando parte de la turísticamente denominada "Riviera Maya", más precisamente localizadas en la península de Yucatán. La relación entre las tres localidades se basa en procesos de ocupación territorial, desarrollos económicos y complementariedad en sus implantaciones que datan de largo tiempo. Jurisdiccionalmente, las tres conformaban el Municipio de Cozumel (1974), siendo actualmente parte de tres administraciones diferentes: el Municipio de Solidaridad (Playa del Carmen, 1993), el Municipio de Tulúm (2008) y el de Cozumel (conformado en 1993).

\subsection{Características generales del territorio y sus bordes urbanos}

La ocupación territorial de Cozumel estuvo condicionada históricamente por la implantación de las actividades productivas y los grupos 
sociales, que conllevan una clara voluntad de segregación. La construcción del poder político y económico del grupo dominante, va a evidenciarse históricamente en la ocupación del suelo y la construcción de la imagen urbana resultante y se exacerba, más recientemente, a través de la modificación del patrón de desarrollo debido a la actividad turística. Esto se acompaña por un sostenido crecimiento poblacional, que comienza a evidenciar como problemática el alojamiento, por lo general periférico, de los sectores populares, que mayoritariamente sostienen dichas actividades.

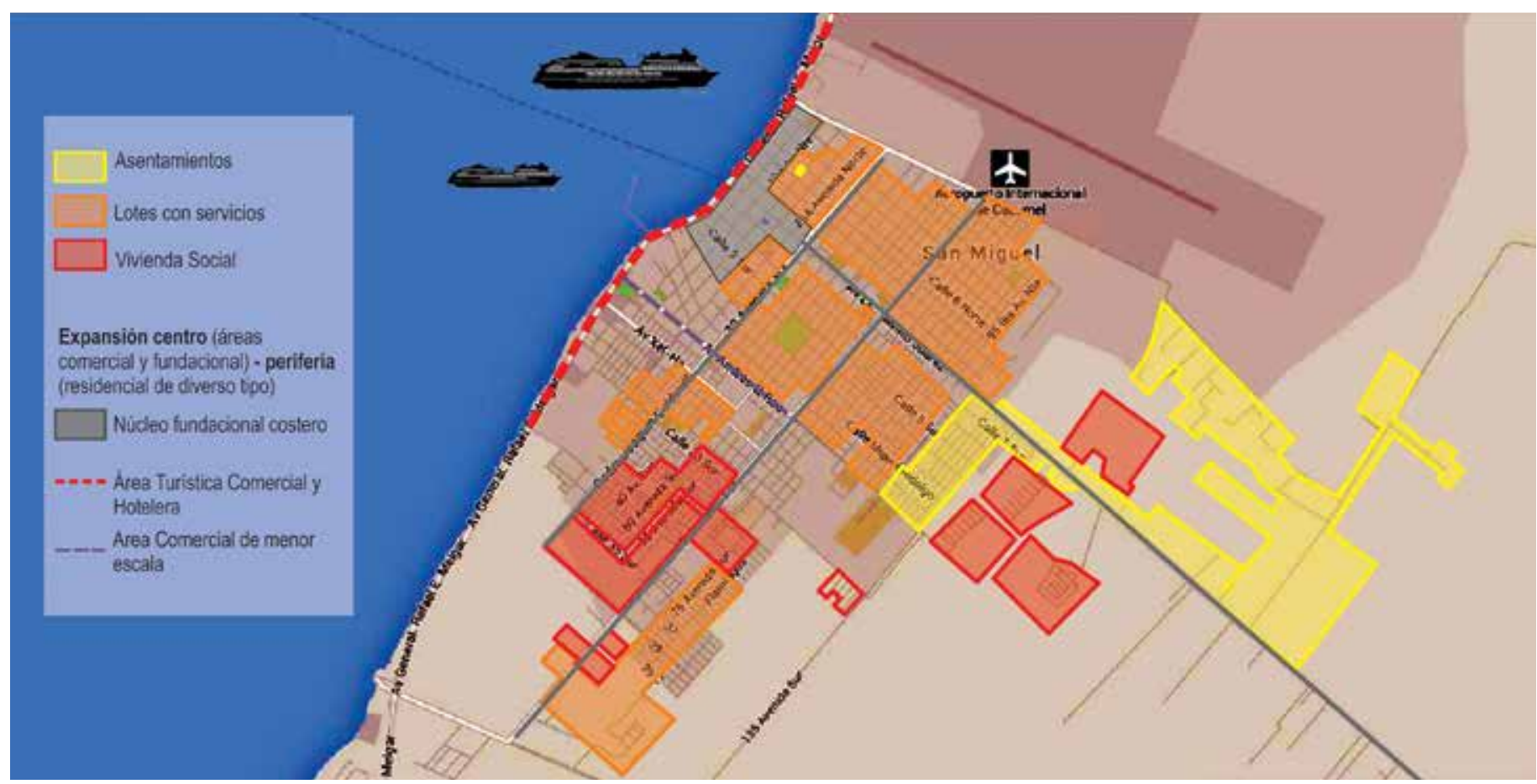

Playa del Carmen, por su parte, posee un desarrollo territorial de origen casi espontáneo, ligado a la implantación de pescadores en el área. Es a partir de la influencia de Cancún —sobre todo en torno a los años ochenta- que comienza a consolidarse como destino turístico, evidenciándose en los últimos años un crecimiento exponencial tanto de su población como de la extensión de la mancha urbana. Entre los factores de este fenómeno, se considera "la migración como una de las causas más importantes, particularmente en las zonas de alto desarrollo turístico, la que se produce por los requerimientos de mano de obra de la industria turística" (Campos, 2011).

Tulúm reviste toda la importancia que su implantación prehispánica evidencia pero también se reconoce un proceso reciente de consolidación institucional y desarrollo turístico. En principio formó parte del circuito turístico Cancún al norte y Chichen Itzá al este. Se advierte que la explotación turística más intensiva comienza a partir de 2002, consolidándose junto con toda la Riviera Maya como un sitio de turismo internacional: "Puerto Morelos, Playa del Carmen, Ciudad Chemuyil y Tulúm pasan a convertirse en los asentamientos humanos de miles de inmigrantes de diferentes orígenes, cuadruplicando el número de habitantes en solamente 10 años. Es decir, de 24352 habitantes en 1995 pasan a 120000 en el 2005" (Fraga Berdugo, 2012, p. 56).

La transformación territorial y poblacional del área comienza en torno a la década del cincuenta, aprovechando el cambio de escenario
Figura 2. Localización de los casos de estudio (Cozumel). Fuente: Elaboración propia. 

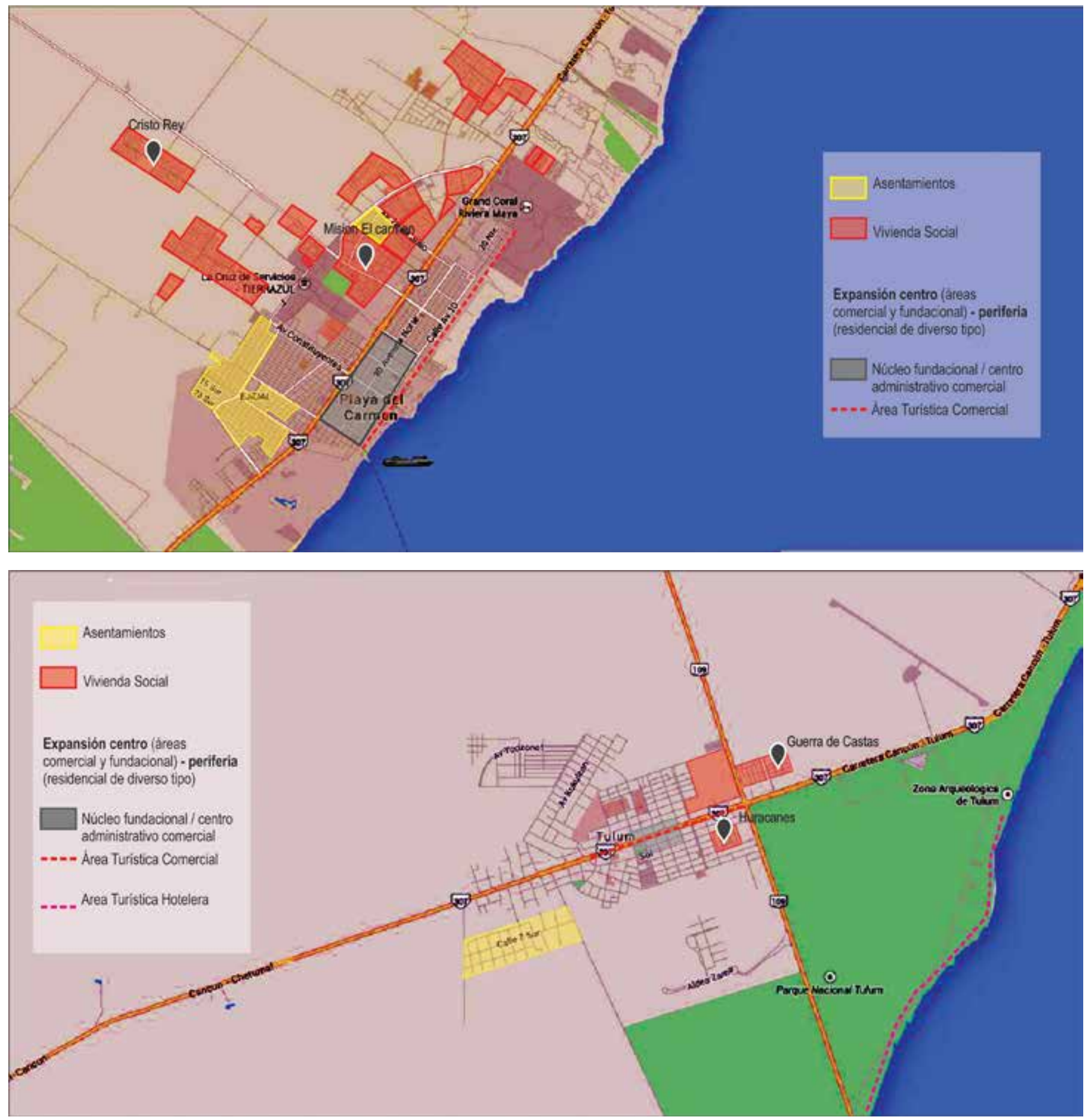

Figura 3 (arriba). Localización de los casos de estudio (Playa del Carmen). Fuente: Elaboración propia.

Figura 4 (abajo). Localización de los casos de estudio (Tulúm). Fuente: Elaboración propia. regional generado a partir de la Revolución Cubana (1959) y sus efectos respecto del turismo norteamericano (Castro, 1988). El surgimiento de Cancún como ciudad planificada y epicentro del desarrollo turístico de la región se constituye en el elemento movilizador del territorio y su transformación.

El desarrollo turístico enunciado genera impactos reconocibles en la configuración de los territorios en cuestión. En este sentido, las particularidades geográficas de cada una de estas localidades repercuten en el modo de ocupación del suelo tanto de las propias actividades vinculadas al turismo cuanto en la residencia de quienes directa o indirectamente sostienen, como población local, su desarrollo.

En este sentido, la lógica casi monocéntrica que presenta Cozumel se organiza en base a la expansión que en torno al núcleo fundacional 
costero se da a partir de crecimientos en cuadrantes y recientemente en anillos de circunvalación que organizan las implantaciones habitacionales masivas. Las actividades turísticas se alojan a uno y otro lado de la isla, definiendo sus bordes, distanciándose de la trama urbana primigenia, casi como enclaves que garantizan el disfrute del paisaje natural (o construido, de los centros hoteleros y recreativos) por sobre la presencia urbana. La única excepción podría constituirse el malecón costero y sus inmediaciones, que concentra casi exclusivamente presencia comercial (plazas) destinada al turismo de cruceros.

En el caso de Playa del Carmen, es una lógica más longitudinal que, a manera de línea de playa, organiza el territorio. El turismo concentra los primeros territorios costeros, mientras que la vivienda popular se localiza en forma paralela hacia el borde interior del territorio, también organizada a través de una serie de viarios que limitan casi fragmentariamente cada una de las colonias residenciales. "A diferencia del resto de las localidades turísticas de la región, la creciente especialización funcional y una relativa segregación se dieron en Playa del Carmen como parte de un proceso de mercado, en donde el área central se reconvirtió hacia el turismo y la población vendió sus terrenos una vez legalizados, trasladándose a las nuevas colonias situadas en la periferia" (Córdoba, García, 2003, p. 125).

En el caso de Tulúm, las actividades vinculadas al turismo masivo -tanto por la presencia de ruinas arqueológicas cuanto por implantaciones de playa, hoteleras o gastronómicas - se separan claramente de la implantación urbana. Sin embargo, la convivencia de actividades turísticas y residenciales se verifica en instancias de un turismo de menor impacto que elige la experiencia de la vida urbana. En este sentido, en la estructura de la localidad conviven las resoluciones de vivienda masiva junto a pequeñas ofertas comerciales, gastronómicas y hoteleras de la ciudad, sin que se conciban bordes periféricos, pero donde claramente se perciben bordes interiores en la trama.

\subsection{La configuración del hábitat y los casos de análisis}

En general, cuando se hace referencia a periferias residenciales en América Latina y el Caribe, señalamos la resultante de la producción social del hábitat. Sin embargo, los mencionados crecimientos poblacionales y las acciones directas recientes, han planteado un fuerte impacto territorial, la producción de vivienda social en las periferias, que acompañan al proceso "autoproducido". En el caso mexicano, la producción habitacional estuvo ligada a la actuación de los gobiernos estatales, durante la últimas décadas del siglo XX, coincidiendo con el período que para el caso de las políticas habitacionales macrodefinidas a escala país, se definían anteriormente como de apoyo al sector constructor (entre 1975 y 1988) y el posterior apogeo de las empresas desarrolladoras de vivienda a partir de 1989.

El desborde en este tipo de propuesta habitacional urbana plantea como punto de partida la desigual en el acceso a movilidad urbana, equipamientos y demás servicios vinculados al derecho a la ciudad en un sentido amplio. Es reconocible la construcción de la ciudad al margen de las lógicas urbanas de la ciudad fundacional o central: la ciudad turística plantea claramente los límites de acceso al suelo para los sectores poblacionales con necesidades habitacionales y que el turismo emplea para los servicios que oferta.

En Cozumel se analizaron situaciones de configuración de hábitat 
diferenciadas y que conforman, en principio, dos bordes urbanos: uno materializado por la presencia física del aeropuerto, configurando el "límite" para la implantación de experiencias espontáneas de ocupación (Las Fincas); y el otro, configurado por la implantación de conjuntos de vivienda social en manos de desarrolladores urbanos (en este caso se analizaron tanto Nueva Generación como las implantadas en el barrio de San Gervasio). En esta localidad también se analizaron casos donde la precariedad habitacional se reconoce como situaciones intersticiales y puntuales dentro de la trama urbana consolidada.

En Playa del Carmen, por su parte, los dos casos analizados presentan configuraciones urbanas diferencias: Misión El Carmen define un tejido característico de la producción de vivienda en parcelas pequeñas y trazado asociado a una idea de ciudad-jardín, mientras que el Fraccionamiento Cristo Rey, localizado en la periferia de la planta urbana sin continuidad con esta, configura un asentamiento (con loteos delimitados regularmente) de baja calidad espacial y material.

Por su parte, Tulúm fue analizado a partir de dos casos puntuales: Huracanes, una implantación residencial de cierta antigüedad que mantiene aún su grado de desconexión manifiesto con el resto de la trama; mientras que Guerra de Castas, siendo una urbanización reciente implantada a la vera de un viario de conexión con el territorio, también evidencia dicha desarticulación, configurando, por otra parte, un tejido urbano de baja densidad, cuyo agregado edilicio es compacto y de reducidas dimensiones.

\section{Dimensiones e indicadores para el análisis}

El análisis de la sustentabilidad en las diversas manifestaciones del hábitat residencial requiere el reconocimiento de dichos indicadores respecto de cada dimensión, atendiendo asimismo a las fuentes de información mencionadas. En este artículo, se toma uno de los objetivos de la Agenda Hábitat, para estructurar la presentación, seleccionando aquellos indicadores que correspondan para cada dimensión.

El objetivo priorizado es de promover el derecho a una vivienda adecuada. El mismo, es enunciado por el Pacto Internacional de Derechos Económicos, Sociales y Culturales cuando "reconocen el derecho de toda persona a un nivel de vida adecuado para sí y su familia, incluso alimentación, vestido y vivienda adecuados, y a una mejora continua de las condiciones de existencia" (ONU DESC, 1976). En este marco, las Observaciones Generales (ONU, 1991) reconocen específicamente el derecho a la vivienda adecuada, identificando una serie de aspectos que lo evidencian: la seguridad jurídica de la tenencia, la disponibilidad de servicios, materiales, facilidades e infraestructura; el hecho de que los gastos relativos a la vivienda sean soportables; la habitabilidad; la asequibilidad; el lugar, orientado al acceso al empleo y servicios básicos; y la adecuación cultural.

Los indicadores que permiten reconocer la concreción de este objetivo (o su carencia) en el contexto de análisis, fueron considerados a partir de los parámetros ONU como de las incorporaciones de otros provenientes de trabajos previos de los equipos universitarios participantes, cuanto de las consideraciones institucionales que en materia de sustentabilidad y hábitat se reconoce en los respectivos países (MINCYT-CONACYT, 2013). 

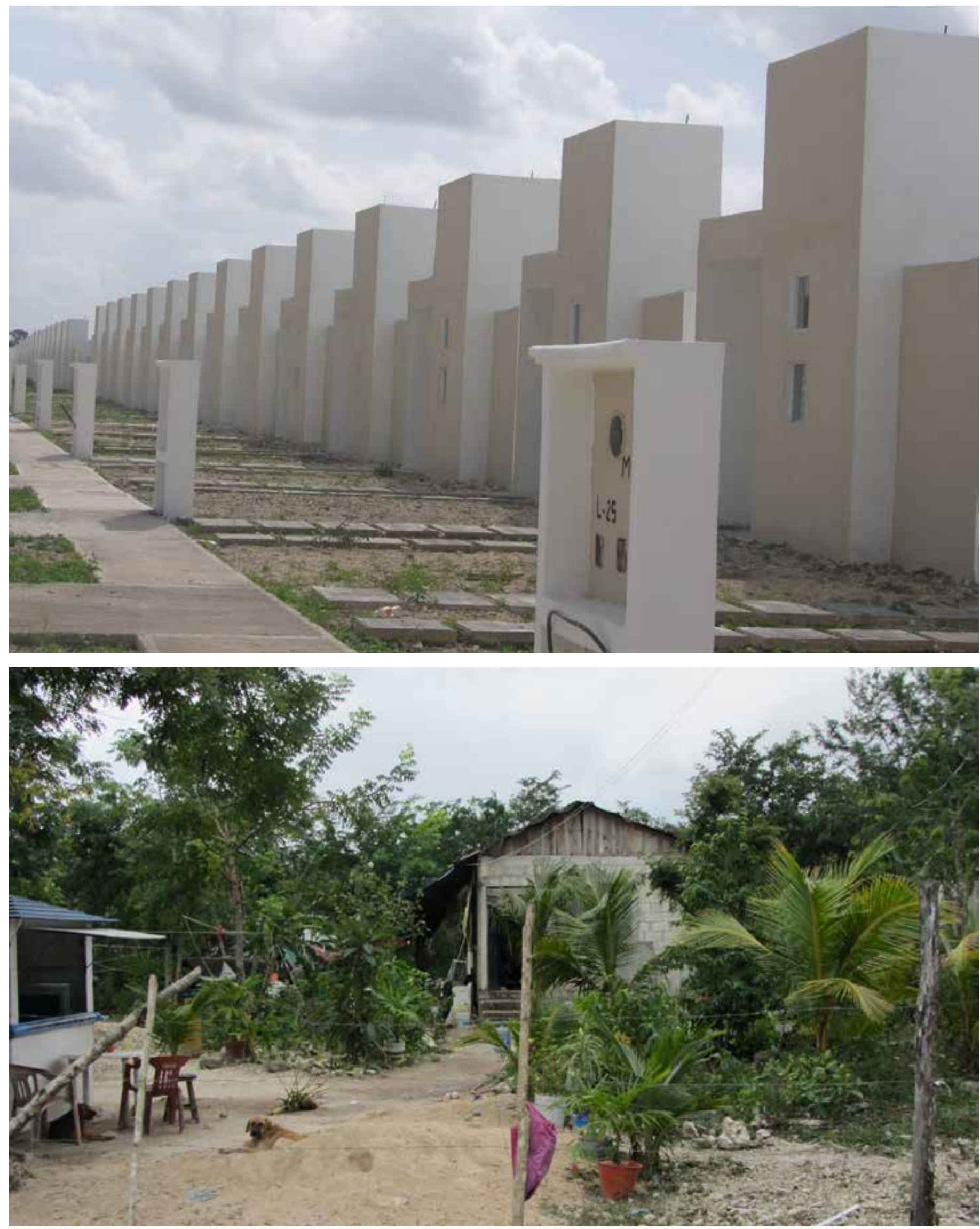

Figura 5. Nueva Generación (Cozumel). Fuente: Elaboración propia.

Figura 6. Las Fincas (Cozumel). Fuente: Elaboración propia. 


\subsection{Dimensión ambiental-urbano-arquitectónica}

\subsubsection{Indicador: Estructuras durables}

La Agenda Hábitat refiere a este indicador como la "proporción de hogares que viven en una casa considerada "durable", es decir, construida en un área sin riesgos y con una estructura permanente y lo suficientemente adecuada como para proteger a sus habitantes de inclemencias del tiempo tales como la lluvia, el calor, el frío y la humedad" (ONU, 1996). En un contexto de alta exposición al riesgo como el que la Riviera Maya encierra, la reincidente acción de huracanes y fenómenos similares, permite considerar la dimensión de la "durabilidad" bajo parámetros diferenciados, atendiendo tanto a la capacidad de respuesta de las estructuras habitacionales ante la ocurrencia de dichos fenómenos cuanto a su capacidad de resiliencia posterior.

El reconocimiento físico de la implantación habitacional atiende a recabar inicialmente datos que remitan a la definición de Agenda Hábitat: la lectura técnica como espacio para el análisis y la comprensión de la manifestación física del contenedor habitacional básico. En el caso de las implantaciones de vivienda social en Cozumel, Playa del Carmen y Tulúm, se reconocen ciertas características básicas respecto de la permanencia de sus estructuras (construcción con materiales tradicionales, predominio de la ejecución por empresa, antigüedad relativamente reciente) que, sin embargo, no siempre revisten características adecuadas respecto de otros aspectos, entre ellos los climáticos. Las particularidades del Caribe evidencian en los casos relevados y su materialización, el insuficiente resguardo ante la lluvia, la inexistencia de espacios intermedios o las inadecuadas situaciones de acondicionamiento ambiental. Por otra parte, estas características no se complementan con la implantación urbana de las viviendas, ya que a la inversa de lo que una zona de selva presupone, carece de la presencia de verde que amortigüe las condiciones ambientales. Por el contrario, esta situación se torna inversa en Las Fincas y el Fraccionamiento Cristo Rey, donde las condiciones de materialidad son más precarias, incompletas y de mayor vulnerabilidad, pero, por el contrario, se verifican situaciones de aprovechamiento y adecuación ambientales propias del sitio en el que se implantan, que evidencian, asimismo, una mayor utilización del espacio abierto con la presencia de hamacas, galerías e incluso actividades específicas como la cocina.

Respecto de la exposición a eventos vinculados al riesgo de huracanes al que se expone la zona, no ha afectado en profundidad las estructuras preexistentes en los casos de Tulúm, ni en El Carmen (Playa) o en Nueva Generación y San Gervasio. Sin embargo, estos hechos, junto con inundaciones o grandes tormentas, son evidenciados por algunos habitantes de asentamientos precarios como la causa habitual que afectó mayoritariamente sus viviendas, en algunos casos, en forma total.

\subsubsection{Indicador: Adecuación cultural}

El planteo inicial respecto de este indicador remite a que "la manera en que se construye la vivienda, los materiales de construcción utilizados y las políticas en que se apoyan deben permitir adecuadamente la expresión de la identidad cultural y la diversidad de la vivienda" (ONU, 1996), siendo abordado con mayor especificidad, apelando a 


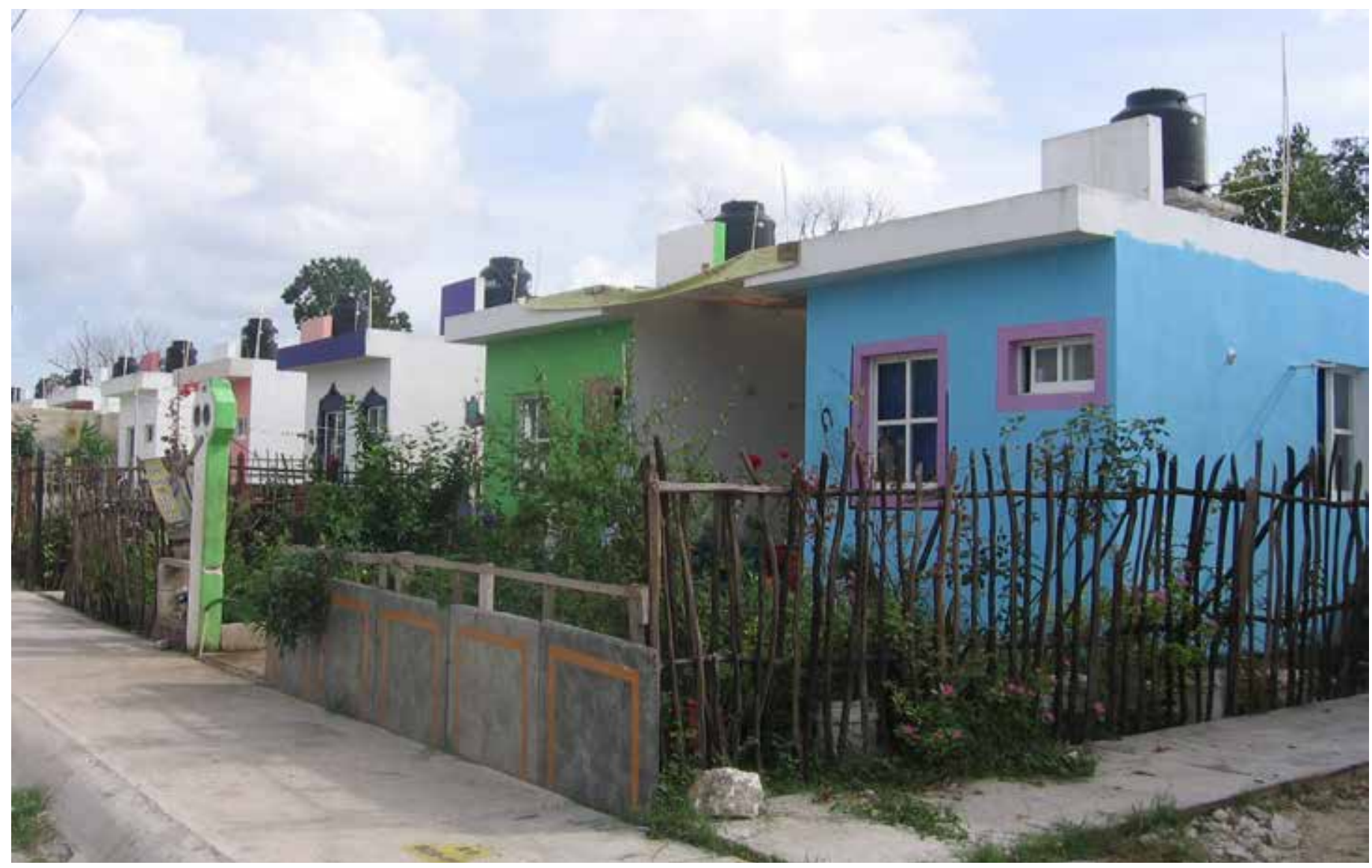

la idea de que la vivienda tienda a "viabilizar la expresión cultural y la diversidad de los distintos individuos y grupos que la habitan" (Rolnik, 2011), valorando los procesos colectivos ligados a la producción de vivienda.

Si bien la implantación residencial generada por las políticas públicas a manera de espacios habitacionales multiplicados en una homogénea periferia urbana indica, en términos generales, que el grado de inadecuación de los mencionados proyectos resulta evidente, cabe destacar, sin embargo, la presencia de la impronta cultural (popular) que se visualiza en las modificaciones exteriores realizadas a la vivienda, una vez que la misma es ocupada por sus habitantes: la aplicación de color, la definición de ciertos espacios intermedios, la presencia de jardines o pequeños espacios verdes en la vivienda, etc. Los mismos son verificables con mayor presencia en los casos donde tanto la antigüedad como la exigua definición proyectual resulta más evidente (ej., las viviendas en San Gervasio o las manifestaciones de autoconstrucción de Las Fincas o Cristo Rey). Las intervenciones sociales buscan reforzar las individualidades, destacar la presencia, a través de la diferenciación cromática, pero también recuperando esos "espacios intermedios" tan caros a la lógica local de definición residencial (ya sea como espacio verde y/o recreativo o como sitio que evidencia la idea de "vivienda productiva") mexicana.

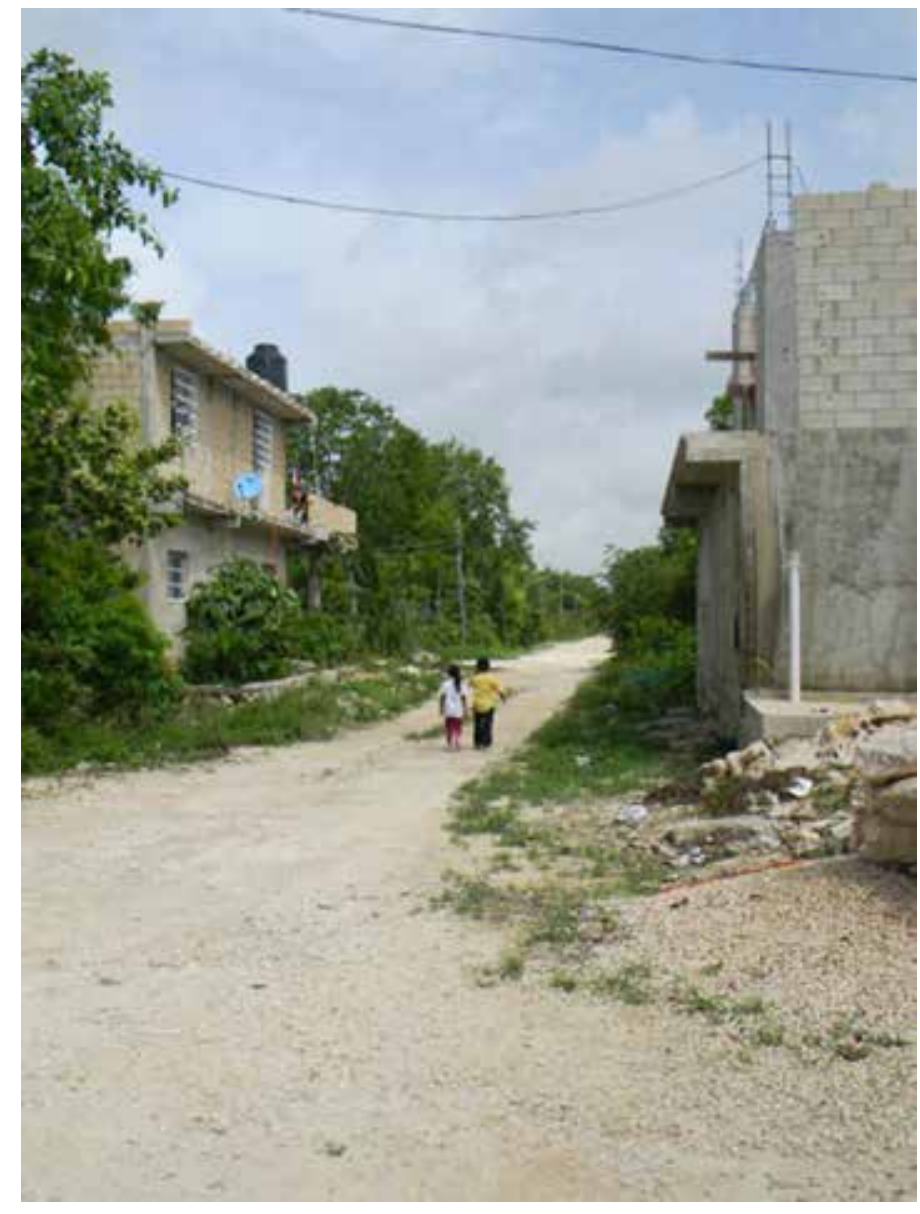

Figuras 7 y 8 . San Gervasio (Cozumel). Fuente: Elaboración propia. 


\subsection{Dimensión social}

\subsubsection{Indicador: Área suficiente para vivir}

Para Agenda 21, se trata de un indicador clave que mide la adecuación a la necesidad humana básica de abrigo, estableciendo esa relación entre la superficie disponible en la vivienda y cantidad de habitantes en la misma: "se considera que una casa proporciona un área suficiente para que sus miembros vivan si no más de dos personas comparten la misma habitación" (ONU, 1996). Esta instancia se traduce en los datos censales como hacinamiento cuando más de tres personas ocupan un dormitorio. En Cozumel, el índice más comprometido respecto de la habitabilidad de las respuestas habitacionales resulta precisamente el hacinamiento. El promedio de ocupantes por vivienda, si bien se encuentra en disminución, es alto: de 1970 a 2005 se pasa de 5,5 a 3,8 habitantes por vivienda aproximadamente (Municipio de Cozumel, 2006, p. 26). La reducción del mismo fue uno de los objetivos evidenciados al momento de encarar las más recientes acciones habitacionales en el marco de encargos gestionados y ejecutados por desarrolladores privados.

En otro sentido, vale destacar la necesidad de evidenciar las particularidades que el término "suficiente" reconoce en contextos culturales y grupos poblacionales diferenciados, entendido como la valoración social del grado de adecuación de dicha superficie y el reconocimiento técnico de la mencionada suficiencia. En este sentido, los relevamientos realizados permiten inferir una situación que visibiliza esta relación entre "superficie adecuada" que el dato numérico indica y su materialización o valoración en el contexto familiar de cada unidad.

En Cozumel, el tipo predominante en las intervenciones recientes es la vivienda unifamiliar, con escalas diferenciadas de respuestas,

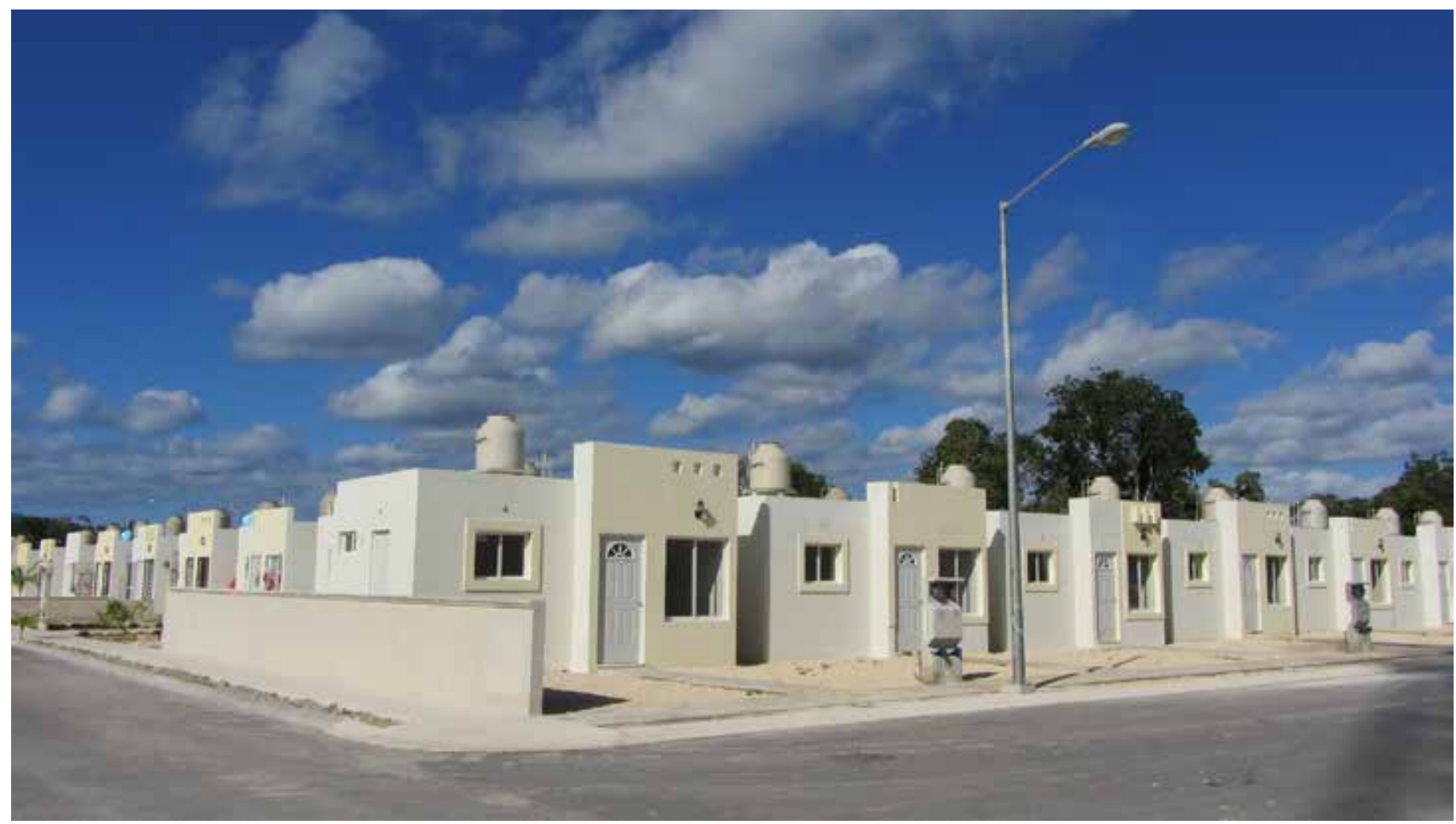




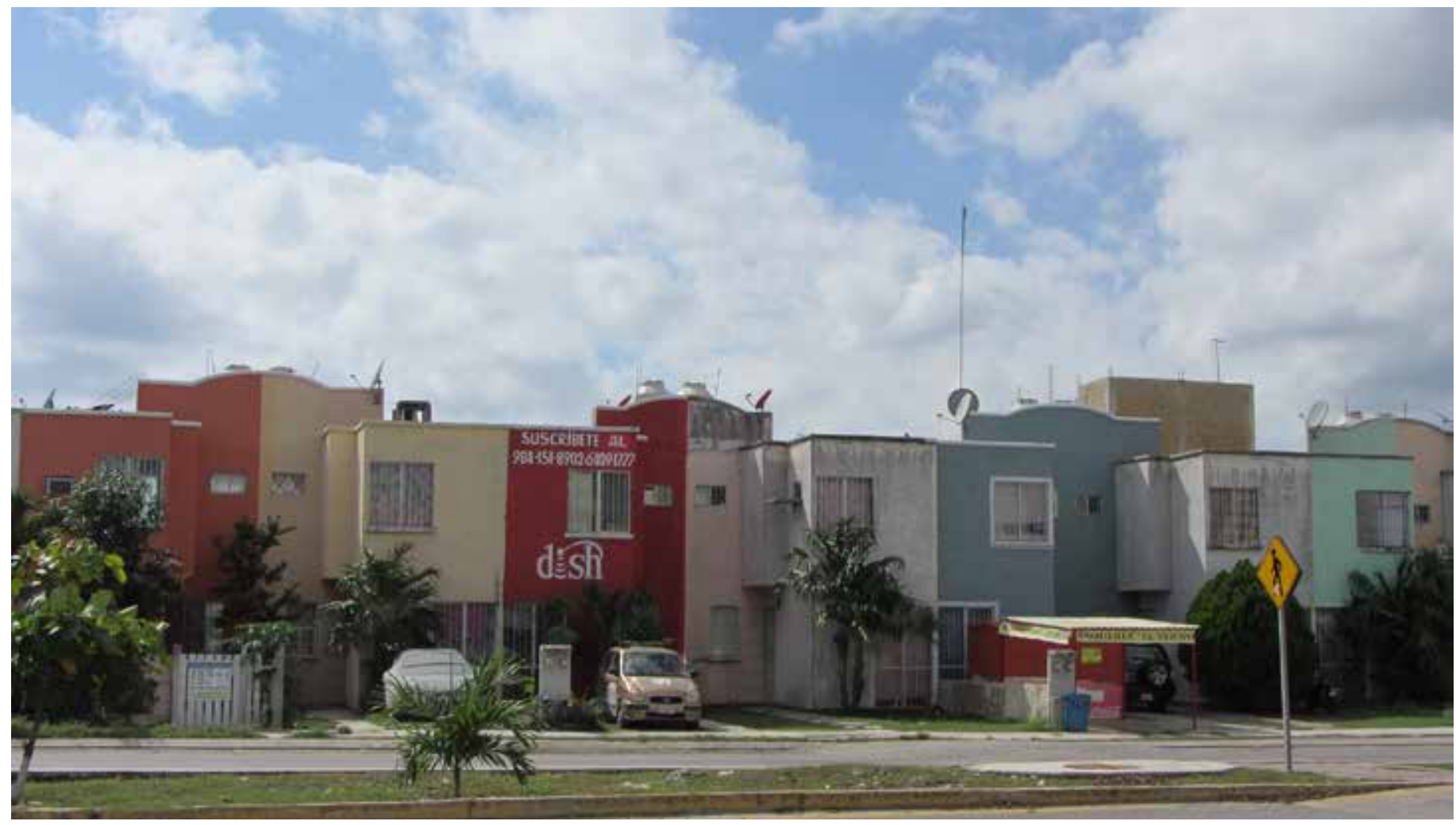

que van desde tipos resueltos en parcelas de escasos $6 \mathrm{~m}$ a 7,50 de ancho (San Gervasio), con superficies que se definen entre 100 y $150 \mathrm{~m}^{2}$ (Nueva Generación) y cuya área construida corresponde a rangos de entre 40 y $50 \mathrm{~m}^{2}$. La vivienda "progresiva" que definió la modalidad tradicional de ocupación de los lotes con servicios en la isla se ve reducida a la "vivienda llave en mano" entregada por el desarrollador, siendo las mencionadas superficies determinantes de la imposibilidad de desarrollar procesos de completamiento y/o ampliación a cargo de los destinatarios.

La misma tipología se reconoce en los casos analizados en Playa del Carmen, apareciendo situaciones tal vez más extremas en Tulúm, donde Huracanes y Guerra de Castas enfatizan soluciones habitacionales lineales de aproximadamente 35 a $40 \mathrm{~m}^{2}$ en exiguas parcelas de poco más de $130 \mathrm{~m}^{2}$.

Por el contrario, en el caso de las manifestaciones residenciales autoconstruidas, se verifican superficies más holgadas que albergan por lo general a familias numerosas y, en ciertos casos, ensambladas. La implantación suburbana por lo general y la ausencia de esquemas de aprovechamiento exacerbado del suelo, dan cuenta de superficies más amplias también en la configuración parcelaria.

En este sentido, resulta significativa la utilización de los espacios que se manifiesta en las viviendas, ya sea generando usos - habitualmente interiores - hacia el exterior, como aprovechando la superficie de las habitaciones con la presencia de hamacas que "multiplican" el área disponible.
Figura 10. San Gervasio (Cozumel). Fuente: Elaboración propia. 


\subsection{Dimensión económica}

\subsubsection{Indicador: Precio de vivienda}

El acceso al suelo urbano, a los servicios y a la posibilidad de adquirir o construir una vivienda, forma parte de la intención de evaluar las condiciones de asequibilidad que se plantean en el contexto mexicano. La idea de vivienda "llave en mano", de vivienda como producto que en su carácter objetual remite a la "solución habitacional" habitual planteada desde las políticas públicas vigentes, implementadas mayoritariamente a partir de la tercerización de las mismas en manos de desarrolladores urbanos.

En este sentido, las respuestas en torno a las posibilidades económicas de acceso a las políticas habitacionales indican esperas prolongadas, cuotas elevadas y ausencia de alternativas tanto habitacionales como de las propias modalidades de gestión. Por otra parte, la implantación periférica y la carencia de equipamientos urbanos significativos condicionan la sostenibilidad económica de las nuevas implantaciones, dependiendo de condiciones de movilidad inadecuadas o de escaso alcance para el conjunto de la población.

Es notoria, asimismo, la presencia de irregularidades respecto del dominio y/o de las condiciones de acceso al suelo por parte de las experiencias de autoproducción del hábitat. En general, estas operaciones, a diferencia de manifestaciones similares en Latinoamérica, no son resultado de ocupaciones informales y espontáneas, sino que resultan movilizadas por desarrolladoras inmobiliarias que adquieren los predios y comercializan los lotes, no carentes de "ilegalidad" en sus operatorias.

El pago de "impuestos prediales" en áreas no reconocidas por catastros o registros urbanos formales da cuenta de una serie de condiciones adversas para la regularización dominial de sus habitantes. En estos casos, resulta significativa la ausencia de servicios y/o el pago elevado de los mismos, incluyendo, asimismo, en este último, la propia extensión de las redes.

\subsection{Dimensión institucional}

\subsubsection{Indicador: El derecho a la vivienda}

Este indicador resulta fundamental al momento de abordar problemáticas habitacionales y el impacto de las políticas que definen su intervención, conceptualizar las implicancias de la noción de vivienda y el ejercicio del derecho a la ciudad. El derecho a una vivienda digna y adecuada, reconocido como universal y amparado bajo los derechos humanos, reconoce que "toda persona tiene derecho a un nivel de vida adecuado que le asegure, así como a su familia, la salud y el bienestar, y en especial la alimentación, el vestido, la vivienda, la asistencia médica y los servicios sociales necesarios" (Declaración Universal DDHH).

En México, la vivienda entendida como derecho ciudadano es reconocido constitucionalmente en su artículo cuarto, junto con otra serie de derechos como la igualdad de género y la libertad de reproducción. Sin embargo, esta garantía constitucional no se verifica en un escenario donde dicho derecho humano se encuentra mediado fuertemente por el accionar del mercado. 
Enrique Ortiz afirma que la vivienda posee dos tipos de aproximaciones bien diferenciadas: como producto o como proceso. El primero "responde al concepto de resolver las necesidades habitacionales de una familia o persona, pero, por ser un bien costoso, implica una producción limitada y atiende a sectores medios y altos. En cambio, como proceso responde a la práctica social, a la forma en que la mayor parte de la gente produce su vivienda de acuerdo con la dinámica de sus recursos, posibilidades, necesidades y sueños" (Ortiz, 2007).

Estas nociones subyacentes en la idea de vivienda predominan en los casos de estudio, atendiendo a configurar mayoritariamente "productos" por sobre "procesos", e incluyen, asimismo, una marcada idea de ciudad, su construcción y acceso. Las implantaciones urbanas generadas por desarrolladores privados evidencian la ausencia de condiciones básicas y cualificadas de urbanidad, así como el hecho de que estos emplazamientos se localizan en situaciones periféricas, una configuración urbana más próxima a la idea del anónimo suburbio residencial que no se constituye en un fragmento de ciudad sino en una suerte de "aglomeración de viviendas".

\section{Conclusiones}

La vivienda como partícipe del proceso de construcción urbana se evidencia en el contexto de estudio, como una clara operación fragmentaria, carente de capacidad de sutura y articulación con la ciudad preexistente. La ciudad generada por desarrolladores urbanos, así como la autoproducida por el conjunto social, carecen de las condiciones de sustentabilidad que implican el equilibrio entre las diversas dimensiones mencionadas, planteándose, en todo caso, una diferenciación en términos de los rasgos predominantes en uno y otro caso.

Las ciudades turísticas mexicanas, particularmente a partir de la injerencia de los desarrolladores urbanos en la cuestión de la vivienda, marcan un punto de inflexión en lo referente al acceso a la vivienda en condiciones de equidad. El acceso al suelo urbano resulta clave, constituyéndose una manifestación de los conflictos vinculados a una construcción histórica del territorio, siendo muchas veces las mismas estrategias de producción de vivienda social las que, en términos de uso del suelo urbano e incidencia en su valor, acentúan la mencionada inequidad. Estas ciudades, pero tal vez con más énfasis Cozumel y Playa del Carmen, evidencian de modo cada vez más contundente la fragmentación o la separación entre el "área turística" —o, en todo caso, capaz de ser mostrada en términos de imagen deseada de la paradisíaca rivera- y aquellos sectores que, en su desplazamiento y exclusión, evidencian esa otra manifestación urbana que alberga a quienes sostienen los servicios de la ciudad "turística". El mercado del suelo juega acá con otros patrones y variables atendiendo a las fluctuaciones -internas y externas- que un destino turístico privilegiado, como el caso de la isla mexicana, plantea con respecto a su construcción y desarrollo, y que la vivienda, en sus diversas manifestaciones, evidencia. El reconocimiento de la vivienda como un derecho ciudadano y, en ese marco, como un paso inicial para el básico ejercicio del derecho a la ciudad, se constituye en una deuda aún pendiente en el contexto del desborde turístico mexicano. 


\section{Referencias bibliográficas}

Bagnera, P., Frausto, O. y Soijet, M. (2012). Indicadores de sustentabilidad aplicados al monitoreo del hábitat urbano: área metropolitana Santa Fe-Paraná, Argentina y Riviera Maya-Cozumel, México. En XXXV Encuentro Internacional RNIU 2012: Paisaje urbano: debate, desafios y sustentabilidad. San Luis Potosí, México.

Bagnera, P., Pennisi, M.B., Frausto, O. y Perdigón, T. (2012). Las políticas habitacionales y su huella urbano territorial: lecturas comparadas de Santa Fe, Argentina y Cozumel, México. En Coloquio Transformaciones Territoriales, San Miguel de Tucumán, Argentina.

Bagnera, P., Ponce, S. y Rojas, J. (2013). La vivienda social y el acceso al suelo urbano en escenarios de "desarrollo" turístico. El caso del Caribe mexicano. En Congreso Nacional de Vivienda. México.

Campos Cámara, B. (2001). Presión turística y urbanística: vulnerables al cambio climático en el Caribe mexicano. Quivera, vol. 13, UNAM.

Córdoba, J. y García, A. (2003). Turismo, globalización y medioambiente en el Caribe mexicano, en Inves- tigaciones Geográficas. Boletín del Instituto de Geografia, 52, UNAM, México.

Fraga Berdugo, J. (2012). Mundo maya. En Guardado, García y Godas (comp.s) (2012). Turismo, globalización y sociedades locales en la península de Yucatán, México, n. ${ }^{\circ}$ 7. El Sauzal, Tenerife, España: Colección Pasos.

Municipio de Cozumel (2006). Cuaderno Estadístico Municipal. Cozumel.

ONU (1991). Observación general 4, El derecho a una vivienda adecuada. En Aplicación del Pacto Internacional de los Derechos Económicos, Sociales y Culturales.

ONU (1996). Agenda Hábitat. Conferencia Hábitat Estambul.

ONU HABITAT (2011). ¿Qué es una vivienda adecuada?. En Relatoría Hábitat Naciones Unidas. Recuperado el 15 de septiembre de 2015, de: http:/ / direitoamoradia.org/?page_id=46\&lang=es.

Ortiz E. (2007). Integración de un sistema de instrumentos de apoyo a la producción social de vivienda. México: HIC AL.

BAGNERA, Paola, y Belén PENISSI. La vivienda y la sustentabilidad en la Riviera Maya: los desbordes del turismo. Hábitat y Sociedad, 2015, n. ${ }^{\circ}$, pp. 97-112.

$<$ www.habitatysociedad.us.es $>$ http://dx.doi.org/10.12795/HabitatySociedad.2015.i8.05

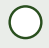

